\title{
Klebsiella pneumoniae in Breast Milk-A Cause of Sepsis in Neonate
}

\section{Pawlik Dorota1, Agnieszka Chmielarczyk², Lorenc Katarzyna1, Michalski Piotr ${ }^{3}$, Lauterbach Jan³, Radziszewska Renata ${ }^{1}$ and Wójkowska-Mach Jadwiga2*}

${ }^{1}$ Clinic of Neonatology, Jagiellonian University Medical College, Krakow, Poland

${ }^{2}$ Deapartment of Microbiology, Jagiellonian University Medical College, Krakow, Poland

${ }^{3}$ Medical Center Ujastek, Krakow, Poland

*Corresponding author: Wójkowska-Mach Jadwiga, Deapartment of Microbiology, Jagiellonian University Medical College, Krakow, Poland, Tel: 48126336033; E-mail: mbmach@cyf-kr.edu.pl

Received date: January 17, 2017; Accepted date: January 24, 2017; Published date: January 31, 2017

Citation: Dorota P, Chmielarczyk A, Katarzyna L, Piotr M, Lauterbach J, et al. Klebsiella pneumoniae in Breast Milk-A Cause of Sepsis in Neonate. Arch Med. 2017, 9:1

Copyright: (C) 2017 Dorota P, et al. This is an open-access article distributed under the terms of the Creative Commons Attribution License, which permits unrestricted use, distribution, and reproduction in any medium, provided the original author and source are credited.

\section{Abstract}

Objective: The aim of the analysis was to present two cases of a late onset sepsis (LOS) caused by Klebsiella pneumoniae (KLE) recognized in prematurely delivered siblings, born within the interval of one year.

Study Design and Result: It was a retrospective study. The first child was born after 32 weeks of pregnancy and the gestational age of the second infant was 29 weeks of pregnancy and died in the course of sepsis without positive blood culture examination. In the second child, sepsis with the same clinical signs was diagnosed, the strains of KLE were isolated from the blood and from the mother's breast milk: the strains were identical, confirmed with PFGE.

Conclusion: Presented results suggest that the source of infections in both siblings was contaminated breast milk. The targeted therapy used in the mother allowed breastfeeding the child.

\section{Keywords: Breast milk; Klebsiella pneumonia; Neonatal} sepsis

\section{Introduction}

The cause of nosocomial infections occurring in patients of neonatal intensive care units are mostly Gram-positive bacteria, and these are often coagulase-negative Staphylococci. Infections caused by Gram-negative bacteria are less common but they are much more severe and more likely to cause death in the course of sepsis. It is believed that about $8 \%$ of this type of infections is caused by Klebsiella pneumoniae (KLP). In preterm infants with very low birth weight in the course of infections caused by KLP the mortality rate can reach $30 \%$.

Until recently it was believed that Streptococcus agalactiae is present in breast milk and may cause sepsis, apart from other pathogenic microorganisms. However, the incidence of such infections has only been reported occasionally. There are several documented cases of infections caused by various microorganisms transmitted through breast milk. Human milk has the potential of carrying infectious agents, which may cause late-onset sepsis. Breast milk transmission of such bacteria like Staphylococcus aureus [1], Streptococcus group B [2], Listeria sp. [3], E.coli [4], KLP [5] has been documented. Breast milk can be considered as a potential source of infection in newborns with recurrent infections or in the case of infection occurring simultaneously in siblings [6]. However, breast milk is the optimum source of nutrition for all children, including preterm and sick neonatal patients.

In this article we present two cases of premature infants with the occurrence of KLP in the gastrointestinal tract. The bacteria were isolated also from the blood of one infant, who was diagnosed with late-onset sepsis. The source of the infection was a breast milk of its mother, in which KLP was found.

\section{Materials}

An embedded case study with two levels of analysis was conducted in April 2011 with retrospective data: 2009. We analyzed clinical and microbiological data with respect to the results obtained in genotyping methods.

Studied strains: In four samples obtained from the mother and infants KLP was isolated from the child's blood culture and the perianal swab culture of the child (sample collection date: 22.04.2011); from the breast milk and the perianal swab culture of the mother (sample collection date: 29.04.2011).

Research susceptibility and genetic typing by PFGE: In the Department of Microbiology of the Jagiellonian University Medical College, disc diffusion method was used to determine drug susceptibility. We used the following discs (Oxoid, Basingstoke, UK) with an antibiotic or chemotherapeutic ampicillin, amoxicillin with clavulanic acid, piperacillin, piperacillin-tazobactam, cefepime, cefotaxime, ceftazidime, 
cefuroxime, cefoperazone with sulbactam, meropenem, aztreonam, ciprofloxacin, amikacin, gentamicin, netilmicin, tobramycin, tigecycline, trimethoprim-sulfamethoxazole. Bacterial isolates were subjected to molecular typing by the pulse-field gel electrophoresis method using a previously published protocol [7]. On the basis of the separation a dendrogram was made in the Gel Compar Applied Maths, using the Dice coefficient and UPGMA algorithm (Unweighted Pair Group Method with an arithmetic mean).

\section{Results}

An infant born as a first child was delivered vaginally after 32 weeks of pregnancy with birth weight of $1650 \mathrm{~g}$; Apgar score evaluated after 1 minute of life was 9 points. In the delivery room nCPAP support was applied, and continued for 10 consecutive days in the NICU. On the grounds of negative tests evaluating concentrations of inflammatory markers (plasma Creactive protein concentration and plasma procalcitonin levels), and negative results of blood culture the congenital sepsis was excluded. The infant was fed enterally from the first day of life with the increasing amounts of breast milk. Also, partial parenteral nutrition was used from the first day of life.

On the 14th day of life there was a sudden deterioration in clinical condition of the patient. Because of increasing respiratory failure the child required intubation and mechanical ventilation. Laboratory studies showed an increase in the following plasma inflammatory markers concentrations: CRP $67.9 \mathrm{mg} / \mathrm{l}$, and PCT $193.2 \mathrm{ng} / \mathrm{ml}$ ). Blood culture evaluation revealed the presence of coagulase-negative Staphylococcus (CNS). However, in the bacteriological examination of stool, KLP was isolated. There was a severe disorder in coagulation test observed (thrombocytopenia, low activity of antithrombin $<30 \%$, prolonged PT and aPTT tests). Infant needed either transfusions of fresh frozen plasma, or platelets. Due to anemia the concentrated red blood cells were transfused. The patient was treated with antibiotics (vancomycin, meropenem), an antifungal agent (Diflukan) was given as the prevention of fungal infections. Because of leukopenia, (white blood cell count $<3000 / \mathrm{mm}^{3}$ ) leukocyte-stimulating factor (Neupogen) was introduced. The infant needed the use of catecholamines (dopamine, dobutamine) and hydrocortisone, because of a significant reduction in blood pressure was observed. Due to decreased antithrombin activity, a formulation containing this factor was transfused. Despite intensive anti-shock, antibacterial and anti-inflammatory treatment, hypotension occurred which consequences were abnormal perfusion and increasing metabolic acidosis. On the $3^{\text {rd }}$ day from the onset of illness the child died with symptoms of septic shock.

An infant born as a second child was delivered vaginally after 29 weeks of pregnancy within the interval of one year after the previous, first child. Its birth weight was $1340 \mathrm{~g}$; Apgar score evaluated in the first minute of life was 8 points, and 10 points on the third minute. Because of the symptoms of respiratory distress syndrome and increased oxygen requirement, a value of $\mathrm{FiO}_{2}>0.35$, the patient obtained one dose of surfactant by the INSURE method and then was switched to noninvasive respiratory support nCPAP system for a period of 7 days. The results of biochemical analyses of blood, obtained within 6-12 hours after birth, showed normal concentrations of inflammatory markers (CRP and procalcitonin). Also, the result of blood culture was negative. Hemodynamically significant patent ductus arteriosus observed within the first three days of life was closed pharmacologically on the $5^{\text {th }}$ day after birth. On the $15^{\text {th }}$ day after birth there was a sudden deterioration of the infant's clinical condition. A decrease in muscle tone and apnea were observed. Also, abdominal distention, and the occurrence of loose stools without any traces of blood were found. Because of the aggravation of the respiratory insufficiency the child was intubated and mechanical ventilation was introduced. Laboratory tests revealed increased plasma CRP concentration (>90 $\mathrm{mg} / \mathrm{L}$ ) and significantly elevated plasma levels of procalcitonin $(12.21 \mathrm{ng} / \mathrm{ml})$. Blood culture was positive and KLP was isolated. Also, in the stool sample, the same bacterium was isolated. Treatment with two antibiotics was introduced according to the results of susceptibility of pathogen (Meropenem and Netromycine). In addition to the antibiotic treatment, formulation of immunoglobulin (Pentaglobin) was added and because of leukopenia, leukocyte-stimulating factor (Neupogen) was administrated.

In addition, during the treatment of sepsis, the patient required a single transfusion of red blood cells and fresh frozen plasma. Within the four consecutive days the child's condition was stabilized. The immune disorders were suspected in the infant because of lymphopenia and a decrease in the absolute value of CD4 T-lymphocytes were found. During hospitalization, the child was fed enterally with breast milk from the first day of life. The milk was stored in a fridge at $4^{\circ} \mathrm{C}$ for up to 24 hours, or was frozen and stored at $-20^{\circ} \mathrm{C}$. Then it was thawed and heated to $25^{\circ} \mathrm{C}-37^{\circ} \mathrm{C}$ and given to the child in a maximum of 4 hours from the start of defrosting. After the mother was discharged, the milk expressed at home was transported with a fridge or thermos. The mother was given instructions on the hospital rules for dealing with human milk and lactation equipment.

Genetic typing of microorganisms was carried out during hospitalization. Following the results indicating that the bacteria present in the breast milk, as well as those that caused the illness in the child belonged to the same epidemic clone, the mother was treated with antibiotics, given probiotics and submitted recommendations for personal and hand hygiene. Lactation was withheld only during antibiotics administration, but it was not stopped. After 7 days of therapy with antibiotics, the mother started to feed her infant with breast milk. The patient was discharged home and was fed exclusively with breast milk.

\section{The microbiological analysis}

All four isolates showed sensitivity to the whole set of antibiotics tested, except for penicillin. The results of genetic typing indicate that all four tested isolates (numbers 6-9) have an identical band pattern, so they belong to the same clone (A). Other isolates of KLE from a previous multicenter study within the Polish Neonatal Network [7] were added to the genetic typing as strains that are material for comparison. It was shown that three of them [3-5] have different, unique restriction 
patterns, and two [1,2] are identical but different from the isolates tested (Figures $\mathbf{1}$ and 2).

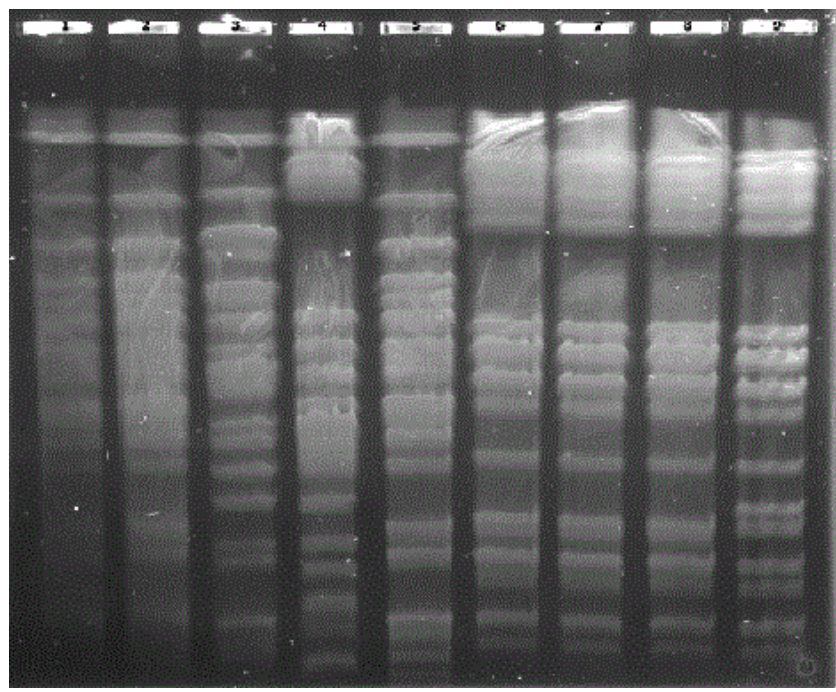

Figure 1 Electrophoresis analysis of KLP.

PFGE-Xbal

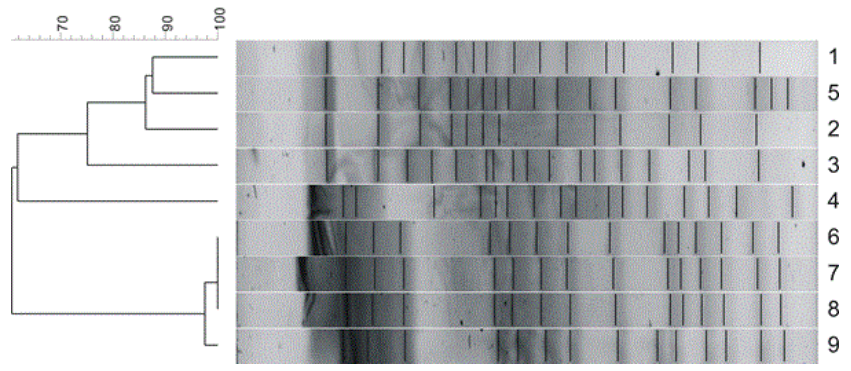

Figure 2 The dendrogram from the electrophoresis of KLP created in Molecular Analyst (Dice coefficient, UPGMA, Tol. $2 \%$ Opt $0.5 \% 0.0 \%$ Low area). From 1 to 5 -strains of KLP from the neonatal network (previous microbiological examination, multicenter, 6-KLP strain, breast milk; 7-KLP strain, rectal swab from the mother; 8-KLP strain, blood, newborn II; 9-KLP strain, rectal swab, newborn II.

\section{Discussion}

Until recently it was thought that breast milk is free of bacteria and the neonatal digestive tract does not contain bacterial flora until birth. The results obtained from the recent studies suggest the occurrence of bacteria in the digestive tract also during the intrauterine life. The presence of the bacterium was detected in the fetal meconium and probably on the skin and, also in the colostrum and breast milk immediately after birth [8]. Currently, it is believed that breast milk is an important source of probiotic bacteria, which colonize the digestive tract immediately after birth. Moreover, it is known that their nature and composition change during the lactation process, which is influenced by many factors [9]. These include maternal nutritional status, obesity, and type of delivery: caesarean section vs. vaginal delivery. Based on the results of recent studies, it can be concluded that the bacteria found in breast milk are not contaminations and what is important, they are the first source of colonization of the digestive tract after birth. There might be specific mechanisms in the mother, which are responsible for contaminated the breast milk with these bacteria that are commensal in the state of good health. On the basis of the results obtained from the experimental studies, it is suggested that dendritic cells found in lymphoid tissue (GALT: gut associated lymphoid tissue) penetrate the intestinal epithelium and "transport" commensal bacteria from the intestinal lumen. These bacteria enter the systemic circulation and finally reach the mammary glands. The opponents of this theory suggest that during labor and delivery the infant's gastrointestinal tract is colonized with the bacteria located in the mother's lower GI tract and the mother's perineal. Then the bacteria localized in the newborn's mouth contaminated the breast while feeding. Based on the speculation described above, we cannot clearly confirm or reject any of these theories. If the described newborns were born by cesarean section, the concept concerning the participation of dendritic cells in the colonization of bacteria in the breast would be more likely. KLP found in the mother's digestive tract was identical with the strain found in the breast milk and in the stools in of both infants and was the cause of sepsis in one of them. The same pathogen was probably also the cause of sepsis in the first child who died in the course of the infection. It may be considered true, based on the clinical symptoms and identical course of illness found in sepsis. CNS found in the blood of the first child, usually does not cause such severe sepsis, but KLE gram-negative bacteria produces lipopolysaccharide endotoxin that may translocate from the intestine into the bloodstream and result in severe clinical manifestations of sepsis, even without the presence of the bacteria in the blood. Unfortunately, this could not be confirmed by strain typing because no KLE strain isolated from the patient was available.

As it has been shown in the experimental studies, KLP has a special "ability" to leak damaging the digestive tract [10]. Its translocation from the gastrointestinal tract into the breast through lymph and blood seems to be possible. Although breast milk has mechanisms that inhibit the inflammatory reactions in the gastrointestinal tract, it must be assumed that the constant "supply" of the pathogenic bacteria might stimulate a pronounced inflammation of the intestinal wall which causes leakage and consequently bacterial translocation.

The most important information that comes from the description of these cases is common practice, aimed at maintaining continuous feeding of the infant with its mother's breast milk. The targeted therapy used in the mother allowed to eradicate the etiological agent of infection from breast milk, and continue breast-feeding the child for a period of the following next several months. It did not result in another episode of sepsis. What should be emphasized, that after applying antibiotics to the mother, the pathogen was eradicated and a control bacteriological test did not reveal KLP presence in the stool. It does not, however, exempt the mother from following 
specific rules of personal hygiene and using probiotics during the lactation period.

In terms of multifactorial beneficial effects of the mother's breast milk on the health of a human being, not only during early development but also in adults that the clinical management described above may suggest the pattern to follow in similar clinical situations.

\section{References}

1. Gastelum DT, Dassey D, Mascola L, Yasuda LM (2005) Transmission of community-associated methicillin-resistant Staphylococcus aureus from breast milk in the neonatal intensive care unit. Pediatr Infect Dis J 24: 1122-1124.

2. Gagneur A, Héry-Arnaud G, Croly-Labourdette S, Gremmo-Feger $\mathrm{G}$, Vallet $\mathrm{S}$, et al. (2009) Infected breast milk associated with lateonset and recurrent group B streptococcal infection in neonatal twins: a genetic analysis. Eur J Pediatr 168: 1155-1158.

3. Svabić-Vlahović M, Pantić D, Pavićić M, Bryner JH (1988) Transmission of Listeria monocytogenes from mother's milk to her baby and to puppies. Lancet 19: 1201.

4. Widger J, O'Connell NH, Stack T (2010) Breast milk causing neonatal sepsis and death. Clin Microbiol Infect 16: 1796-1798.
5. Donowitz LG, Marsik FJ, Fisher KA, Wenzel RP (1981) Contaminated breast milk: a source of Klebsiella bacteremia in a newborn intensive care unit. Reviews of Infectious Diseases 3: 716-720.

6. Godambe S, Shah PS, Shah V (2005) Breast milk as a source of late onset neonatal sepsis. Pediatr Infect Dis J 24: 381-382.

7. Wójkowska-Mach J, Chmielarczyk A, Borszewska-Kornacka M, Domańska J, Gadzinowski J, et al. (2013) Enterobacteriaceae infections of very low birth weight infants in Polish neonatal intensive care units: resistance and cross-transmission. Pediatr Infect Dis J 32: 594-598.

8. Madan JC, Salari RC, Saxena D (2012) Gut microbial colonization in premature neonates predicts neonatal sepsis. Arch Dis Child Fetal Neonatal 97: F456-F462.

9. Cabrera-Rubio R, Collado MC, Laitinen K (2012) The human milk microbiome changes over lactation and is shaped by maternal weight and mode delivery. Am J Clin Nutr 96: 544-551.

10. Zhang C, Sherman MO, Prince LS (2012) Paneth cell ablation in the presence of Klebsiella pneumoniae induces necrotizing enterocolitis (NEC)-like injury in the small intestine of immature mice. Dis Model Mech 5: 522-532. 